\title{
Inhibition of OATP1B1 by tyrosine kinase inhibitors: in vitro-in vivo correlations
}

S Hu, R H J Mathijssen, P de Bruijn, S D Baker and A Sparreboom

Correction to: British Journal of Cancer (2014) 110, 894-898. doi:10.1038/bjc.2013.811; published online 7 January 2014

After publication of the above paper in the British Journal of Cancer, a discrepancy was found in the text. The Results section contains the following: 'As a representative of this class of TKIs, sorafenib was further evaluated and found to also potently inhibit the OATP1B1-mediated transport of docetaxel with a halfinhibitory maximum concentration of $6.96 \mathrm{~nm}$ (Figure 1B)...' However, Figure 1B reports the concentration as $69.6 \mathrm{nM}$.

The authors would like to apologise for this error. The correct concentration is $6.96 \mathrm{~nm}$. 